\title{
Especialización, tecnología y crecimiento en el modelo Ricardiano
}

\author{
Gabriel Porcile \\ Departamento de Economia, Universidade Federal do Paraná \\ e Investigador del CNPq, Brasil \\ Marcio Holland \\ Fundação Getúlio Vargas, Universidade Federal de Uberlândia \\ e Investigador del CNPq, Brasil \\ Mario Cimoli \\ CEPAL, Santiago de Chile y Universidad de Venecia \\ Luciana Rosas \\ Departamento de Economia, Universidade Federal do Paraná, Brasil
}

\section{Palabras llave}

Modelo Ricardiano, crecimiento económico, convergencia y divergencia internacionales.

\section{Classificacion JEL F43}

Key words

Ricardian model, economic growth, international convergence and divergence.

JEL Classification F43

\section{Resumen}

En este artículo se propone un modelo Norte-Sur que permite estudiar cómo los cambios en la brecha tecnológica afectan la especialización y el crecimiento en la economía del Sur. La contribución del artículo consiste en proponer una nueva especificación para la influencia de la brecha sobre la especialización. Además, se discuten las implicaciones de la dinámica de la brecha para el análisis de los procesos de convergencia y divergencia internacionales, en el marco de un modelo ricardiano de comercio con un continuo de bienes. Posteriormente, se testean las predicciones del modelo por medio de un análisis de panel de los determinantes del crecimiento económico en los años noventa. Para ello, se utilizan en el trabajo empírico indicadores recientemente propuestos por la literatura, tanto para la variable tecnológica (la dimensión schumpeteriana del modelo) como para la que refleja el dinamismo del patrón de especialización (dimensión keynesiana).

\begin{abstract}
The paper presents a North-South trade model that discusses how changes in the technology gap affect specialization and growth in the Southern economy. The contribution of the paper is to propose a new specification for the influence of the technology gap on the pattern of specialization. It also analyzes the implications of the dynamics of the technology gap for the process of international convergence and divergence, within the context of a Ricardian trade model with a continuum of goods. Subsequently, the predictions of the model are tested through a panel analysis of the determinants of economic growth in the nineties. With this objective, new proxies recently proposed by the literature for the technological variable (the Schumpeterian dimension of the model) and for the dynamism of the pattern of specialization (the Keynesian dimension) were included in the empirical work.
\end{abstract}




\section{Introducción}

En este artículo se propone un modelo Norte-Sur que permite estudiar cómo los cambios en la brecha tecnológica afectan la especialización y el crecimiento en la economía del Sur. Se toma como punto de partida los trabajos de Cimoli (1988) y Dosi, Pavitt y Soete (1990), quienes combinan el modelo ricardiano de especialización y comercio con el modelo keynesiano de crecimiento con restricción de balanza de pagos. La contribución del artículo consiste en proponer una nueva especificación para la influencia de la brecha sobre la especialización, y en discutir sus implicaciones para el análisis de los procesos de convergencia $\mathrm{y}$ divergencia internacionales.

Posteriormente, se testean las predicciones del modelo por medio de un análisis de panel de los determinantes del crecimiento económico en los años noventa (1990-99). Estos años representan un período en que la mayor parte de las economías latinoamericanas ensaya políticas de rápida liberalización comercial, sin que al mismo tiempo se diseñen políticas de catching-up tecnológico. En la medida que la hipótesis del modelo sea correcta (la brecha tecnológica afecta el patrón de especialización y el crecimiento relativo de los países en la economía internacional), la ausencia de una política tecnológica más activa podría explicar, por lo menos en parte, el bajo dinamismo que mostró la región en el período.

En el estudio se utilizan indicadores recientemente propuestos por la literatura, tanto para la variable tecnológica (dimensión schumpeteriana) como para la que refleja el dinamismo del patrón de especialización (dimensión keynesiana). Para la primera, se utiliza el índice ArCo de capacidades tecnológicas, que permite realizar comparaciones entre las capacidades tecnológicas de los distintos países en distintos momentos del tiempo (Archibugi y Coco, 2003). Por otro lado, los factores asociados al comportamiento de la demanda internacional en la ecuación del crecimiento son captados a través de la participación de los sectores dinámicos (alta tecnología) en la estructura de las exportaciones de cada país. Esta participación se obtuvo a partir de los datos disponibles en el World

Development Indicators.

El trabajo está organizado en tres secciones, además de la introducción y de los comentarios finales. En la 
primera sección se presenta una breve revisión de la literatura sobre los vínculos entre tecnología y crecimiento, en la segunda sección se presenta el modelo formal y en tercera sección se lo estima para los años noventa.

\section{1_Tecnología y crecimiento}

Existe un cierto consenso entre quienes estudian el crecimiento económico en torno a la idea de que la tasa de aumento de la productividad es clave para definir la tasa de crecimiento de largo plazo del producto interno bruto de una cierta economía (Fagerberg, 2000). Más aún, la mayor parte de los economistas tiende a concordar que la fuerza motriz por tras del aumento de la productividad es el aprendizaje tecnológico - la innovación y la difusión de tecnología hacia el conjunto del sistema económico (Fagerberg, 1994; Nelson and Pack, 1997; Verspagen, 1993). Así, las marcadas diferencias que se observan en la economía internacional en términos de niveles de ingreso per capita y de bienestar entre países, está asociada a diferencias de productividad que, en última instancia, se explican por asimetrías en las capacidades tecnológicas. La importancia de estas asimetrías es tal, que algunos autores las consideran como la gran línea divisoria entre el mundo desarrollado y el mundo en desarrollo (Arocena y Sutz, 2003).

Al mismo tiempo, la tecnología no sólo afecta la tasa efectiva o potencial de aumento de la productividad, sino también el patrón de especialización, esto es, el número y el tipo de bienes que una cierta economía es capaz de producir competitivamente. Economías muy rezagadas tecnológicamente, y que no han tenido suerte en la "lotería de las commodities”, estarán propensas a tener su crecimiento limitado por la aparición recurrente de desequilibrios externos. En el caso de las economías en desarrollo, existe un vasto potencial inexplorado de recursos, en particular humanos, que la economía no utiliza, porque su escasa capacidad de importar les impone un techo a la inversió y al crecimiento (Prebisch, 1986; FfrenchDavis, 2000; Rodríguez, 2002). Predominan, en este caso, restricciones de demanda, cuya contrapartida es el desempleo, o la persistencia del subempleo en sectores de subsistencia. Estos aspectos (del lado de la oferta y la demanda) están en el centro de lo que podría llamarse la perspectiva keynesiano-schumpeteriana sobre 
especialización y crecimiento, en gran medida convergente con la tradición estructuralista latino-americana. En síntesis, el tipo de relación causal entre las variables sugerido por modelos de este tipo puede ser descrito como sigue:

a. La competitividad auténtica - la capacidad de cada país de mantener o aumentar su participación en los mercados externo e interno, sin reducir el salario real de sus trabajadores (Fajnzylber, 1990) - es una función de la distancia del país con respecto a la frontera tecnológica y de su capacidad de acortar esa distancia rápidamente. Cuando la brecha tecnológica es muy alta el país rezagado sólo tendrá capacidad de producir un número limitado de bienes, aquellos de menor intensidad tecnológica, en condiciones de baja productividad relativa.

b. Como en los sectores de baja intensidad tecnológica la productividad tiende a crecer menos que en los de más alta intensidad tecnológica, el tipo de especialización internacional que se deriva de a) implica también en menor potencial de aprendizaje y de aumento de la productividad en el largo plazo.

c. Si bien no es perfecta, existe una correlación entre intensidad tecnológica y dinamismo de la demanda internacional. Por esta razón, países rezagados tecnológicamente no sólo tienen menos oportunidades de aprendizaje y de innovación, sino que también enfrentan una demanda menos dinámica por los bienes que producen. Como resultado, están expuestos a crisis recurrentes de balanza de pagos, las que reflejan la baja elasticidad ingreso de la demanda de sus exportaciones frente a la alta elasticidad ingreso de la demanda de sus importaciones.

d. El endeudamiento externo, en condiciones favorables de liquidez internacional, puede momentáneamente aliviar la restricción externa, pero ésta reaparece en el largo plazo. Es más, en la medida que los intereses y el principal de la deuda deben pagarse, es posible que la restricción reaparezca de 
forma aún más intensa, dando lugar a ciclos en los que el crecimiento impulsado por la deuda es seguido por un doloroso ajuste externo.

e. El conjunto de los factores enumerados reduce el crecimiento efectivo de las economías periféricas e impide el pleno aprovechamiento de las oportunidades asociadas a los retornos crecientes y al aprendizaje en la inversión y la producción. Se generan así condiciones propicias para un escenario de divergencia internacional, esto es, un escenario en el cual la brecha del ingreso per capita de los países ricos y de los países pobres aumenta de forma persistente, así como la brecha del aprendizaje y de las capacidades tecnológicas.

f. La posibilidad de reducir los salarios relativos en el Sur puede compensar hasta cierto punto el aumento de la brecha, generando un tipo de competitividad espuria. Sin embargo, cuando los diferenciales de productividad son muy elevados o aumentan en el tiempo, una estrategia basada exclusivamente en salarios bajos no puede sostenerse y reduce el bienestar en el largo plazo.

Existen varias maneras posibles de formalizar estas ideas, cada una de ellas con sus ventajas y desventajas. En este trabajo se adopta un cierto camino, el del modelo ricardiano de crecimiento y comercio, que tiene la gran ventaja de considerar, explícitamente, el impacto del progreso técnico sobre la diversificación de la estructura productiva y los cambios en el patrón de especialización. Esto permite analizar, de forma más rigurosa, los vínculos entre la brecha tecnológica y la tasa de crecimiento compatible con el equilibrio externo.

\section{2_Especialización, comercio y crecimiento económico: el modelo ricardiano}

\section{1_Una Introducción al Modelo Ricardiano}

En esta sección se presenta brevemente el modelo ricardiano, originalmente propuesto por Dornbush et al. (1977). Existe en la economía mundial un continuo de bienes $i=0 \ldots n \mathrm{y}$ dos 
países, el Norte (centro) y el Sur (periferia). Se supone, además, que existe un solo factor de producción - el trabajo - y que cada bien $i$ se produce con un número de horas de trabajo igual a $a_{i}$ en el Sur y $a_{1}^{*}$ en el Norte. La variable $A i=a_{1}^{*} / a_{i}$ representa la productividad relativa del Sur con respecto al Norte. Mientras mayor (menor) es $A i$, mayor (menor) es la ventaja comparativa del Sur (Figura 1). Es posible ordenar los bienes $i$ desde cero hasta $n$, en una secuencia creciente con la ventaja comparativa del Norte. De este modo, el bien número cero es aquél para el cual la ventaja comparativa del Sur es máxima, y el bien $n$ es aquél para el cual la ventaja comparativa del Norte es máxima. Se define, además, una variable $z=i / n$, que puede adoptar valores entre 0 y 1 , de manera que el bien $z=1$ será ahora el bien para el cual la ventaja comparativa del Norte es máxima.

\section{Figura 1_El Modelo Ricardiano}

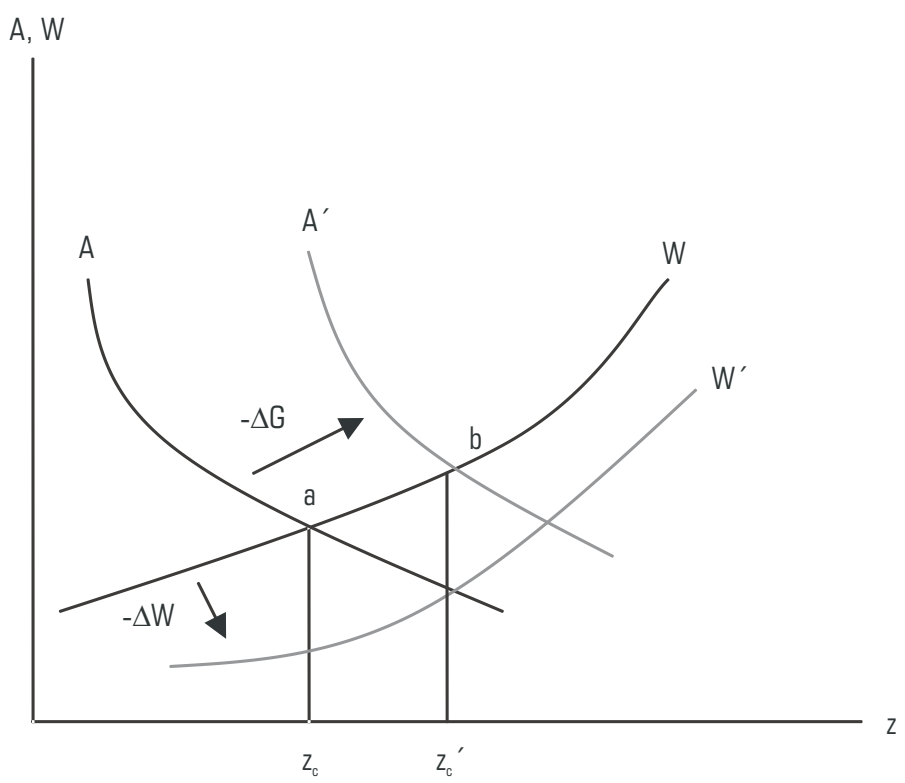

Fuente: Obstfeld e Rodoff (1995, p. 240). 
La variable $W=w / w^{*}$ representa el salario nominal relativo del Sur con respecto al del Norte. Esta relación aumenta con el número de bienes producidos en el Sur, suponiendo que las economías funcionan con pleno empleo, debido al efecto positivo que el aumento de la producción ejerce sobre la demanda de trabajo. Como las dos economías funcionan en condiciones de competencia perfecta y los precios se forman exclusivamente a partir de los costos unitarios del trabajo, el Sur producirá todos aquellos bienes para los cuales $A>W$. Puede verse en la Figura 1 que el Sur produce los bienes de cero hasta $\tau_{c}$, y el Norte produce los bienes restantes $\left(1-z_{c}\right)$.

La posición de la curva $A($ z) depende del nivel de la brecha tecnología Norte-Sur $(G=T n / T s)$, esto es, de la razón entre el acervo de conocimientos acumulados en el Norte (Tn) y el acervo acumulado en el Sur (Ts). Como se ha dicho, la curva $A(z)$ es negativamente inclinada porque fue construida ordenando los bienes de tal forma que los valores de z. más bajos corresponden a aquellos en que la productividad relativa del Sur es más alta. Se asume que a medida que se va hacia la derecha se producen bienes más intensivos en tecnología para los que la productividad relativa del Sur es menor para un mismo valor de la brecha de conocimientos Norte-Sur $(G)$. En otras palabras, dada la brecha tecnológica, las diferencias de productividad son más altas en los bienes de mayor intensidad tecnológica. Adicionalmente, se supone que cambios en la brecha Norte-Sur producen un movimiento proporcional de aumento de la productividad relativa del Sur en la producción de todos los bienes. La reducción de la brecha eleva la eficiencia relativa del Sur en todos los bienes, sin alterar la declividad de $A(z)$. Imagínese por un momento que el Sur reduce la brecha $(-\Delta G$, en la Figura 1$)$ : la curva $A$ se moverá hacia la derecha, hasta alcanzar la posición $A^{\prime}$. Como resultado, el Sur habrá cambiado su patrón de inserción externa, siendo capaz de producir un conjunto mayor de bienes con un salario relativo más alto. Este proceso representa lo que Fajnzylber (1990) denominara un aumento de la competitividad auténtica, o sea, un aumento de la participación del Sur en los mercados externo e interno, acompañado de un aumento de los salarios reales. Inversamente, una reducción del salario relativo $(-\Delta W)$ también permite diversificar la 
estructura productiva, al trasladar hacia abajo la curva $W$, pero ello ocurre a expensas de una reducción del salario real en el Sur (competitividad espuria en la denominación dada por Fajnzylber).

Debe observarse, además, que el impacto de un cambio en el salario relativo sobre la especialización depende de la inclinación de $A$. Si esta inclinación - el multiplicador de la brecha tecnológica (Cimoli, 1988) - no es muy alta, pequeños cambios en el salario relativo producen cambios significativos en la especialización. Inversamente, un alto valor del multiplicador de la brecha (una curva $A A$ muy inclinada) implica que cambios en el salario relativo no tendrán el poder de modificar substancialmente la competitividad externa de la economía.

Cambios en el patrón de especialización producen cambios transitorios en la tasa de crecimiento compatible con el equilibrio externo y reducen la distancia entre los productos del Norte y del Sur. Este tema se aborda en la próxima sección, a través de un modelo sencillo, en que las relaciones entre las variables mencionadas (brecha tecnológica, salarios y crecimiento con equilibrio en la balanza de pagos) son exploradas de forma más rigurosa.

\section{2_ Un modelo de especialización y crecimiento}

El modelo se divide en tres bloques.

El primero describe cómo evoluciona la brecha en el tiempo y cómo se determina la brecha de equilibrio. El segundo combina la información sobre la brecha con la del mercado de trabajo, de tal forma que allí se definen la competitividad y la especialización. El tercer bloque se concentra en las tasas de crecimiento relativas del Norte y del Sur (o sea, la emergencia de procesos de convergencia o divergencia), tanto cuando la brecha esta en equilibrio como durante la dinámica de transición.

\subsection{1_La brecha}

Se supone que la brecha de equilibrio se define a partir de un proceso simple de difusión de tecnología por imitación o catching-up. En este proceso, mientras mayor es la brecha, mayor es la tasa de difusión de tecnología hacia el Sur y más rápido se reduce la distancia entre los polos del sistema Norte-Sur (Fagerberg, 1994). Así:

$$
\hat{G}=\rho-\left[\mu-\mu\left(\frac{T s}{T n}\right)\right]=\rho-\left[\mu-\mu\left(\frac{1}{G}\right)\right]
$$

donde $G=T s / T n$ es la brecha tecnológica y $\hat{G}=\dot{G} / G$ es su tasa de crecimiento en el tiempo, Tn es el 
$\ldots \ldots \ldots \ldots$

1 Una posibilidad sumamente interesante es que el cambio en la estructura productiva realimente la reducción de la brecha. En este caso la variación de $G$ sería no sólo una función de la propia brecha sino del número de bienes z que la economía produce. Se tendría así un sistema con dos variables de estado, la brecha y la estructura productiva. Un análisis sobre el impacto de los retornos crecientes sobre el crecimiento en un modelo de dos sectores puede encontrase en Ros (2000, capítulo 7).

2 El salario real en cada país, sin embargo, puede estar cambiando en función de los cambios en la productividad del trabajo y del precio de los bienes.

3 Una discusión detallada de las implicaciones del modelo de Lewis para la teoría del crecimiento puede ser encontrada en Ros (2000).

En este trabajo se adopta una fórmula especialmente simple del modelo de salarios de Lewis como manera de mantener la atención centrada en la dimensión tecnológica de la competencia internacional. acervo de conocimientos tecnológicos del Norte, Ts el acervo del Sur, $\rho$ es la tasa de progreso técnico exógeno en el Norte y $\mu$ un parámetro positivo que mide el esfuerzo del Sur por imitar las capacidades del Norte. De esta ecuación se obtiene el valor de equilibrio de la brecha tecnológica, que es el que iguala la tasa de crecimiento de la brecha en el tiempo a cero. Formalmente:

$\frac{\dot{G}}{G}=0 \Rightarrow G^{*}=\frac{\mu}{\mu-\rho}$

Así, bajo el sencillo supuesto de una relación lineal directa entre brecha y difusión, la brecha de equilibrio puede ser encontrada como una función exclusivamente de los parámetros que representan el esfuerzo tecnológico en los dos polos.

\subsection{2_ La productividad, los salarios y la diversificación}

Para encontrar la proporción de bienes producidos por la periferia, se parte de la ecuación de productividades relativas, definida como:

$\frac{a^{*}(z)}{a(z)}=A(z)=\alpha-\beta G-b z$

donde $\alpha, b$, y $\beta$ son parámetros positivos. Como la brecha contribuye a determinar el valor del intercepto de la curva $A(z)$, la ecuación (3) explicita el vínculo entre la brecha y la posición de esta curva. Ya fue mencionado que esta representación implica que la reducción de la brecha tecnológica induce un aumento de la productividad relativa del Sur similar en los bienes de baja (por hipótesis, valores más bajos de z) y alta tecnología (valores más altos de z.). ${ }^{1}$ Por otro lado, se asume que los salarios nominales en el Sur y el Norte son constantes, lo que inmediatamente garantiza que el salario relativo Sur-Norte $\left(w / w^{*}\right)$ también lo sea (ecuación 4). ${ }^{2}$ De esta forma la curva $W$ será horizontal y no positivamente inclinada, como fuera representada en la Figura 1.

$\frac{w}{w^{*}}=W=b$

Este supuesto implica admitir que el mercado de trabajo de la periferia funciona à la Lewis, o sea, que hay un excedente de mano de obra en el sector rural o en el sector informal que puede ser rápidamente incorporado a la producción sin cambios en la tasa nominal de salario (siempre y cuando esta tasa represente un salario real superior al producto medio en el sector de subsistencia). ${ }^{3}$ Se asume, también, que el Sur es un país pequeño, y que por lo tanto, la expansión de sus 
exportaciones es incapaz de afectar significativamente el mercado de trabajo en el Norte. ${ }^{4}$ Una forma simple de formalizar esa idea es considerar constantes el empleo y el producto nominal de la economía del Norte, de tal forma que en equilibrio su producto real crece a la misma tasa que la productividad del trabajo $(\rho)$. Dado que existe competencia perfecta en el mercado de bienes, el precio de los bienes producidos en el Norte y en el Sur cae a una tasa igual a sus respectivas tasas de aumento de la productividad.

Bajo estos supuestos, igualando $A(z)=W$, se obtiene el número de bienes producidos en la periferia, $z_{c}$.

$z_{c}=\frac{\alpha-\beta G-b}{b}$

¿Qué valores asume $z_{c}$ cuando la brecha se encuentra estable (o sea, cuando $G=G^{*}$ y por lo tanto $\left.\hat{G}=0\right)$ ? En este caso, la ecuación (5) puede escribirse como:

$z_{c}=\frac{(\alpha-b)(\mu-\rho)-\mu \beta}{(\mu-\rho) b}$

Calculando la derivada parcial de (5’) con relación a $\mu$, se comprueba que un aumento en el esfuerzo de imitación realizado por la periferia (aumento de $\mu$ ) induce un aumento del valor crítico $z_{c} .{ }^{5}$ Inversamente, un aumento de la tasa de innovación en el centro (si no es seguida por un esfuerzo mayor de catching up, esto es, por una respuesta activa de la periferia que redunde en una elevación de $\mu$ ), se traduce en un valor menor de $z_{c}$, la proporción de bienes cuya producción se localiza en la periferia. Finalmente, mientras mayor es la velocidad $b$ con que aumenta el diferencial de productividad a medida que se diversifica la economía, menor será también el valor de $z_{i}$.

\subsection{3_El equilibrio en balanza de pagos}

Se supone ahora que los consumidores gastan un porcentaje exactamente igual de sus ingresos en cada uno de los bienes producidos. $\mathrm{Al}$ hacer este supuesto, el modelo excluye una forma importante a través de la cual la estructura productiva puede afectar el crecimiento, esto es, las diferencias en la elasticidad ingreso que existen entre los bienes. Se mantendrá tal supuesto como forma de obtener curvas de demanda más fácilmente tratables desde el punto de vista formal, pero se lo levantará posteriormente al realizar un ejercicio empírico.

Si la periferia produce hasta el bien $z_{c}$ inclusive, entonces las ventas en el mercado externo (exportaciones del Sur) serán iguales al ingreso nominal del Norte $\left(\gamma^{*}\right)$ multiplicado por $z_{c}$ (Obstfeld y Rogoff, 1996, p. 240). De la misma forma, como la participación de los
4 Como los principales mercados son los del Norte, este supuesto parece razonable. Sin embargo, ello no implica que la economía del Norte no pueda ser afectada por la del Sur en otros aspectos. Como se verá más adelante, en la medida que los bienes del Sur son parte de la cesta de consumo de los trabajadores del Norte, la tasa de inflación en el Sur puede afectar el producto real y el bienestar del Norte.

5 La derivada parcial de (5') con relación a es igual a $\beta \rho /(\mu-\rho)^{2} b$, un número que es, sin ambigüedad, positivo. También es fácil comprobar que la derivada parcial de $z_{c}$ con relación a la brecha de equilibrio $G^{*}$ es igual a $-\beta / b$, lo que confirma la relación inversa entre la brecha de equilibrio y la diversificación productiva del Sur. 
bienes producidos por la periferia en el gasto total de sus propios consumidores es $z_{c}$, las ventas en el mercado interno serán iguales al ingreso nominal del Sur (y) multiplicado por $z_{c}$ y las importaciones serán iguales a ese mismo ingreso multiplicado por $\left(1-z_{c}\right)$.

Con tasa de cambio fija e igual a la unidad, el equilibrio en cuenta corriente exige que $\left(1-z_{c}\right) y=z_{c} y^{*}$.

Si hay entrada líquida de capitales (por ejemplo, en un montante $c$ ), es necesario considerar el equilibrio en la balanza de pagos, que puede escribirse como: $\left(1-z_{c}\right) y=z_{c} y^{*}+c$. La ecuación (6) abajo proporciona el nivel del ingreso nominal en la periferia consistente con el equilibrio externo. Se comprueba que las exportaciones de la periferia dependen del número de bienes que ella misma produce y del ingreso de la economía mundial, mientras que sus importaciones dependen del número de bienes que el centro produce, de su propio ingreso y de la entrada líquida de divisas $(c)$.

$y=\frac{z_{c}}{1-z_{c}} y^{*}+\frac{c}{1-z_{c}}$

Suponiendo $c=0$, substituyendo (5) en (6), y expresando el resultado en términos del nivel del producto nominal relativo Sur-Norte consistente con el equilibrio externo en los dos polos, se obtiene:
$\frac{y}{y^{*}}=\frac{u(G)}{b-u(G)}$

donde $u(G)=\alpha-\beta G-h$. La derivada parcial de $(7)$ con respecto a $G$ es igual a

$\frac{\partial \frac{y}{y^{*}}}{\partial G}=\frac{-b \beta}{[b-u(G)]^{2}}$

La ecuación (8) muestra el impacto negativo del aumento de la brecha tecnológica sobre el ingreso relativo con equilibrio externo. También es fácil ver en (6) que los ingresos nominales del Norte y del Sur solamente serán iguales cuando $z_{c}=1 / 2$, es decir, cuando la producción de los bienes se distribuye por mitades en los dos polos del sistema.

\subsection{4_Convergencia y divergencia}

Si se calcula la derivada de la ecuación (7) (que no considera los movimientos de capital) con relación al tiempo se obtiene:

$\frac{y}{y^{*}}\left(\hat{y}-\hat{y}^{*}\right)=\frac{-b \beta \dot{G}}{\left[(b-u(G)]^{2}\right.}$

Las variables con acento circunflejo indican tasas proporcionales de crecimiento (i.e. $\hat{y}-j / y)$. La ecuación (9) muestra que la tasa de crecimiento del ingreso nominal del Sur 
será menor que la del Norte cuando aumente la brecha tecnológica.

Inversamente, una reducción de la brecha $(\dot{G}<0)$ conlleva a una tasa proporcional de crecimiento mayor del Sur frente a la del Norte $\left[\left(\hat{y}-\hat{y}^{*}\right)>0\right]$.

Una expresión más simple, que ofrece la misma idea en términos de la tasa de crecimiento de z, (la proporción de bienes producidos por la periferia), puede obtenerse rescribiendo la ecuación (6), $\operatorname{con} c=0$, de la siguiente forma:

$\frac{y}{y^{*}}=\frac{z_{c}}{1-z_{c}}$

Derivando (10) con relación al tiempo y reordenado el resultado se obtiene:

$\frac{y}{y^{*}}\left(\hat{y}-\hat{y}^{*}\right)=\frac{\dot{z}_{c}}{\left(1-z_{c}\right)^{2}}$

Multiplicando los dos lados de la ecuación (11) por $\left(y^{*} / y\right)$ :

$\left(\hat{y}-\hat{y}^{*}\right)=\frac{\dot{z}_{c}}{\left(1-z_{c}\right)^{2}}\left(\frac{y^{*}}{y}\right)$

Substituyendo $\left(y^{*} / y\right)$ por la ecuación (10), se llega al siguiente resultado, que explicita la relación entre convergencia y cambios en la estructura productiva:

$\hat{y}-\hat{y}^{*}=\frac{\hat{z}_{c}}{1-z_{c}}$
Naturalmente, interesa sobre todo observar lo que ocurre con los ingresos reales, y no sólo con los ingresos nominales. La ecuación (13) describe la evolución de la diferencia entre las tasas proporcionales del crecimiento del ingreso real del Sur y del Norte, donde $p$ y $p^{*}$ representan los niveles de precios del Sur y del Norte, respectivamente (como siempre, el acento circunflejo indica tasas proporcionales de crecimiento).

Obsérvese, sin embargo, que bajo los supuestos de competencia perfecta y de idénticas cestas de consumo de los trabajadores, la tasa de inflación será la misma en los dos países. Ello implica que la tasa de convergencia del ingreso nominal es la misma que la del ingreso real ( $y_{R}$ en el Sur e $y_{R}^{*}$ en el Norte), como se indica en la ecuación (15).

$\hat{y}_{R}-\hat{y}_{R}^{*}=\frac{\hat{z}_{c}}{1-z}+\left(\hat{p}^{*}-\hat{p}\right)$

$\hat{y}_{R}-\hat{y}_{R}^{*}=\hat{y}-\hat{y}^{*}=\frac{\hat{z}_{c}}{1-z} \operatorname{con}\left(\hat{p}=\hat{p}^{*}\right)$

Así, variaciones de la brecha tienen como resultado directo procesos reales de convergencia o de divergencia internacionales, según sea el signo de esta variación (negativo o positivo, respectivamente). Obsérvese que la ecuación (14) relaciona directamente la 
tasa de convergencia con los cambios en el patrón de especialización del Sur, los que a su vez dependen exclusivamente de los cambios en la brecha.

Los Gráficos 2a-2d permiten visualizar el efecto sobre la brecha, la especialización y el crecimiento de un aumento del esfuerzo de imitación del Sur, que se expresa a través de la elevación del parámetro $\mu$ de la ecuación de difusión de tecnología (ecuación 1).

El Gráfico 2a muestra lo que ocurre en el ámbito tecnológico. El aumento del esfuerzo de aprendizaje (de $\mu_{1}$ para $\mu_{2}$ ) en el Sur provoca un aumento del intercepto y de la inclinación de la curva $\hat{T}_{s}$, con la consiguiente reducción de la brecha de equilibrio, de G1 para G2. Esto a su vez produce un traslado de la curva $\mathrm{AA}$ (que es una función de $\mathrm{G}$, de $\mathrm{AA}_{\mathrm{G} 1}$ para $\mathrm{AA}_{\mathrm{G} 2}$ ) y un aumento del número de bienes producidos en el Sur, de $z_{1}$ para $z_{2}$ (Gráfico 2b). El cambio en el valor de z. implica que ahora el nivel del ingreso nominal con equilibrio externo para el Sur es mayor que antes para cada nivel del producto en el Norte (Gráfico $2 \mathrm{c}$ ). Dado $y^{*}=\bar{y}^{*}$, el nuevo nivel del ingreso nominal en el Sur compatible con el equilibrio externo será $y_{2}>y_{1}$.

Finalmente, el Gráfico 2d muestra como se ajusta el producto real del Sur y del Norte frente a los cambios en la brecha a lo largo del tiempo. Se supone que inicialmente la brecha se encuentra en equilibrio $(\hat{G}=0)$ y por lo tanto $z$ permanece estable, siendo $\hat{y}_{\mathrm{R}}-\hat{y}_{\mathrm{R}}^{*}=0$. En otras palabras, la estabilidad de la brecha implica que las dos economías crecen en términos reales a la misma tasa exógena de crecimiento de la economía del Norte (la tasa $\rho$, que es la inclinación de las curvas logarítmicas de $y_{R}$ e $y_{R}^{*}$ antes del momento To). En To, el Sur aumenta su esfuerzo de aprendizaje, lo que hace que la brecha comience a reducirse y la estructura productiva del Sur a diversificarse. A medida que $G$ se reduce y zaumenta en el tiempo $(\hat{z}>0)$, la tasa de crecimiento del Sur se torna más alta que la del Norte $\left(\hat{\jmath}_{R}-\hat{y}_{R}^{*}>0\right)$ y el producto del Sur se aproxima al del Norte (convergencia). Esta tasa de convergencia se obtiene por medio de un aumento del empleo $L$ en el sector moderno del Sur, $\hat{L}=\hat{q} / 1-$ z. Al mismo tiempo, la economía del Norte muestra un ligero aumento de la tasa de crecimiento real, el que se explica por una reducción de la tasa de inflación (recuérdese que el ingreso nominal no ha cambiado), gracias a la aceleración del progreso técnico en el Sur, y a la correspondiente reducción del precio de los bienes importados. 
Figuras 2a-2d_Efectos del aumento del esfuerzo de aprendizaje en el Sur $(\mu)$

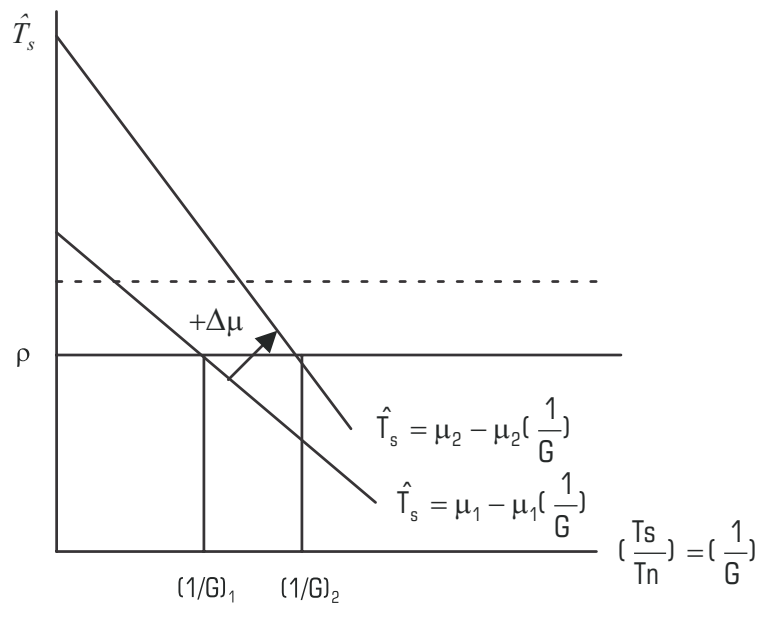

Gráfico 2a

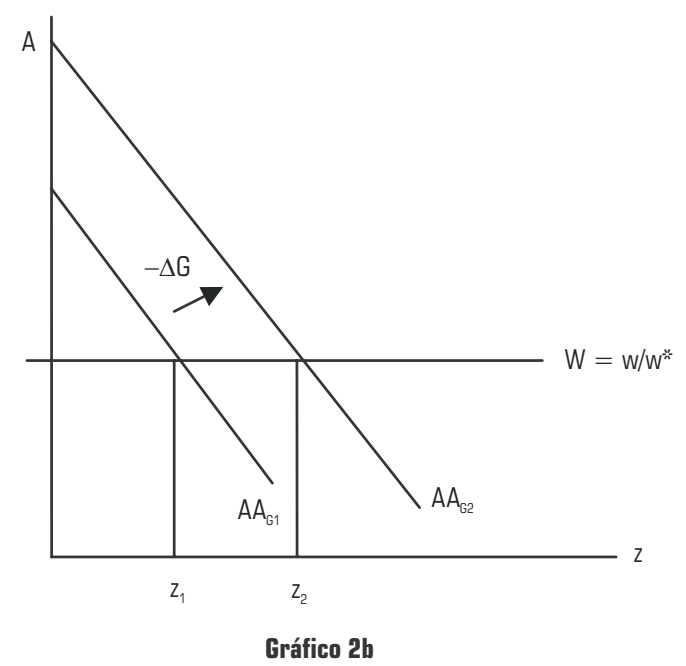

Fuente: Los autores.

nova Economia Belo Horizonte 16 (3) 483-506 setembro-dezembro de 2006

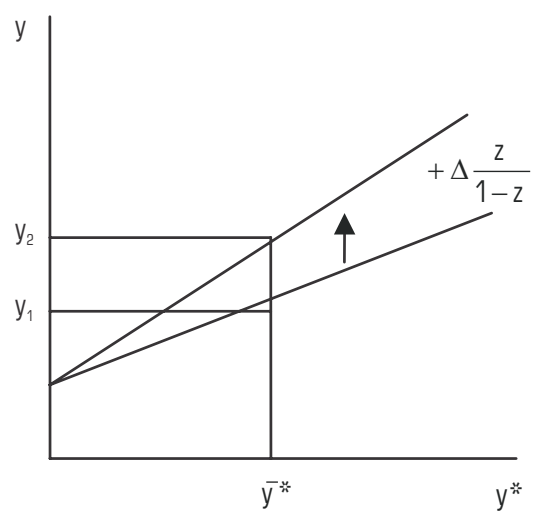

Gráfico 2c

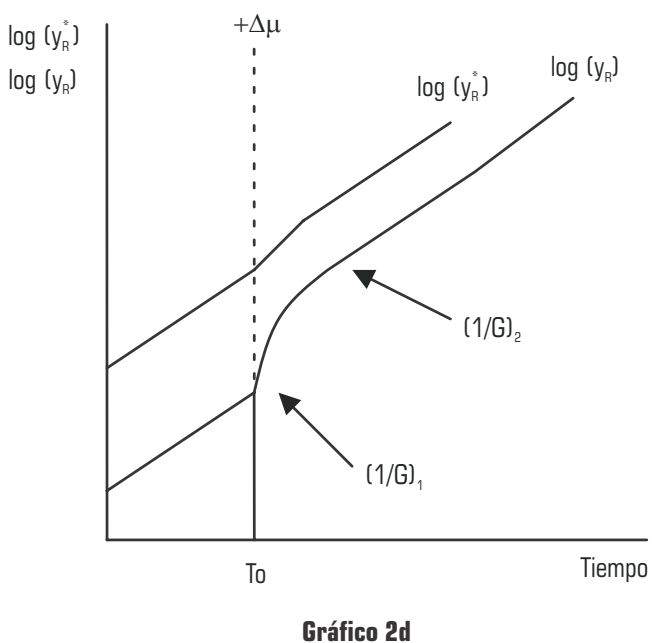


Cuando la brecha se estabiliza nuevamente, también se estabilizan zy la tasa de crecimiento del Sur, que vuelve a ser la misma que la del Norte. Debido al proceso de convergencia, cuando $G$ alcanza su nuevo equilibrio, el Sur ha conseguido reducir la distancia entre su nivel de su producto (nominal y real) con el del Norte. Naturalmente, una reducción del esfuerzo de imitación del Sur tendría consecuencias opuestas: divergencia hacia un nuevo equilibrio con una brecha mayor, tanto en términos de las capacidades tecnológicas como en el nivel del producto.

A seguir, una versión lineal del modelo anterior se estima econométricamente para los años noventa, usando indicadores recientemente propuestos en la literatura para las capacidades tecnológicas y el dinamismo de la demanda mundial por las exportaciones de cada país.

\section{Un test empírico para los años noventa}

La idea de que la tecnología es una variable clave para explicar el comportamiento del comercio y del crecimiento ha sido testada empíricamente de varias formas (ver, por ejemplo, Amendola, Dosi and Papagni, 1993). Uno de los problemas más difíciles de resolver en este tipo de ensayos es encontrar una buena proxy para las capacidades tecnológicas de cada país. Frecuentemente se utiliza el número de patentes obtenidas en los Estados Unidos, un indicador que presenta algunas desventajas cuando se estudia los países en desarrollo. En ellos el progreso técnico asume principalmente la forma de difusión de tecnología, y de innovaciones o adaptaciones adicionales, que muchas veces no pueden ser patentadas.

Por esa razón y sin olvidar que toda tentativa de tornar operacional el concepto de brecha tecnológica se deparará con problemas empíricos difíciles de resolver plenamente, en este trabajo se utiliza un indicador llamado ArCo, recientemente propuesto por Archibugi y Coco (2003). Se trata de un indicador que toma en cuenta un conjunto amplio de variables representativas de las capacidades tecnológicas de cada país (incluyendo, entre ellas, las patentes) y que puede ser estimado tanto para los países desarrollados como para aquellos en desarrollo. Se trata de un indicador que permite, además, realizar 
comparaciones entre las capacidades en distintos momentos del tiempo. Este indicador se construye combinando las siguientes categorías:

i. número de patentes per capita;

ii. publicación de artículos científicos per capita;

iii. cobertura de internet;

iv. cobertura telefónica;

v. consumo de energía eléctrica;

vi. porcentaje de la población en edad universitaria matriculada en cursos superiores de ciencias y de ingeniería;

vii. media de años de escolaridad de la población mayor de 14 años; viii. porcentaje de la población con capacidad de leer y escribir (definida como la capacidad de leer, escribir y entender una sentencia simple sobre la vida cotidiana).

El Gráfico 1 ofrece una primera aproximación a la relación entre ingresos por habitante y capacidades tecnológicas medidas por el ArCo. Se observa una fuerte relación positiva entre ambas variables. Países con un indicador ArCo superior a 0,5 tenían en el año 2000 una media de PIB por habitante superior a los US\$24000, mientras que aquellos con un ArCo inferior a 0,5 mostraban un PIB por habitante de aproximadamente US\$7000. Vale la pena notar también que no hay ningún país latinoamericano con un indicador ArCo superior a 0,5 (el mejor posicionado es Argentina con un índice igual a 0,426$)$, lo que contrasta con los indicadores de los países asiáticos que realizaron un intenso proceso de convergencia en las décadas pasadas como Corea del Sur $(0,607)$ y Taiwán $(0,665)$. También los valores del indicador ArCo son inferiores en América Latina al que muestran algunos países de la periferia europea como Portugal $(0,450)$, Polonia $(0,465)$ y España $(0,516)$. Obsérvese, sin embargo, que la correlación entre PIB por habitante y capacidades tecnológicas, si bien es muy alta, no es perfecta, ya que muchos países disponen de recursos naturales o de formas de inserción externa muy específicas, que les permiten crecer sin que haya una inversión proporcional en conocimientos. Dos ejemplos conspicuos bastan para ilustrar este punto: Kuwait, por un lado, ocupa el lugar 25 en el ranking de países según su PIB por habitante, pero está en el lugar 41 en el ranking del indicador ArCo; Luxemburgo, a su vez, es el primero en el ranking de PIB por habitante, pero sólo obtiene el lugar 27 de acuerdo al indicador ArCo. 
Gráfico 1_Correlación entre crecimiento por habitante y capacidad tecnológica (2002)

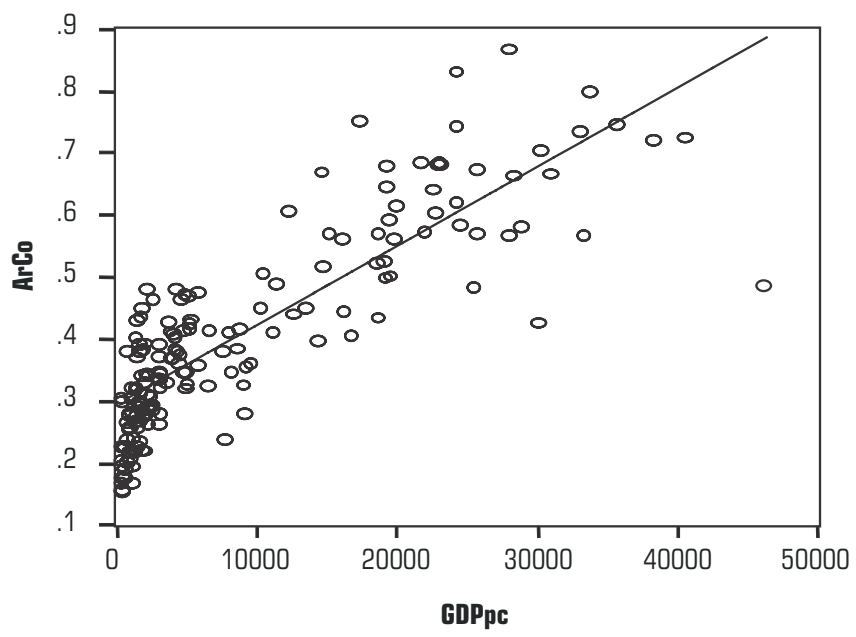

Fuente: World Bank (2006) y Archibugi y Coco (2003).

El modelo de crecimiento con restricción de balanza de pagos sugiere que el efecto de la tecnología se da por medio de sus efectos sobre el patrón de especialización. Ello implica que el indicador ArCo no debe ser incluido directamente en la estimación del modelo, sino como variable instrumental en un proceso de estimación en dos etapas (LS2S).

Adicionalmente, la prueba propuesta en este trabajo también incluye una variable representativa de la dimensión keynesiana del crecimiento. En el sencillo modelo presentado antes, diferencias en el comportamiento de la demanda entre bienes no fueron consideradas, pero tal supuesto no es sustentable empíricamente. Modelos que sólo consideran las asimetrías tecnológicas del lado derecho de la ecuación y que, por lo tanto, desconsideran el papel desempeñado por el dinamismo del patrón de especialización de cada país (por la vía de la restricción en balanza de pagos), estarían omitiendo variables relevantes. Como se sabe, esto tiene como consecuencia la obtención de resultados sesgados y poco confiables en el proceso de estimación. 
Por esa razón, se incluyó en el modelo a ser estimado una proxy del dinamismo de la demanda mundial por los bienes de cada país representada por la participación de los sectores dinámicos en las exportaciones totales del país $(S)$. Se entiende que la especialización en sectores dinámicos es un buen indicador de la capacidad de la economía de entrar en los mercados de más rápido crecimiento y de driblar de esa manera la restricción externa.

Un conjunto amplio de posibles indicadores de especialización productiva puede ser encontrado en el World Development Indicators del Banco Mundial, tales como:

1. exportaciones de productos de alta tecnología en el total de las manufacturas exportadas (HTEXP);

2. exportaciones de manufacturas como porcentaje de las exportaciones totales (MANUF);

3. exportaciones de materias primas agrícolas como porcentaje de las exportaciones totales (AGRI);

4. términos de intercambio (To'T).

Existe una relación empíricamente fuerte entre el nivel tecnológico de un producto y el dinamismo de su demanda. Por eso, se asume que cuanto mayor es el valor del indicador HTEXP, mayor es el dinamismo de la demanda de exportaciones. El mismo argumento es válido para el indicador MANUF, mientras que lo opuesto debe esperarse para el indicador AGRI. Por otro lado, en la medida que una demanda mundial más dinámica tiende a generar una mejora en los términos de intercambio, estos últimos también fueron incluidos en el análisis.

Se observó que la variable HTEXP tiene una media de 12,5\%, pero algunos países obtienen valores tan altos como $73 \%$. Las variables

MANUF y AGRI, en cambio, varían muy poco. El valor mínimo encontrado para MANUF fue de $40 \%$, mientras que AGRI varía solamente entre 0 y 5 $\%$. Por ser muy amplio, el concepto de manufacturas no consigue discriminar correctamente entre países. La intuición sugiere que la variable HTEXP debería captar mejor diferencias en el patrón de especialización. La misma revela una fuerte correlación con la variable ArCo, lo que confirma la relación entre el patrón de especialización y la base tecnológica del país (Gráfico 2).

$\mathrm{El}$ modelo propuesto predice que el patrón de especialización más dinámico debe estar relacionado con una tasa de crecimiento más alta. 
"

Los valores de las reservas en meses de importaciones fueron también obtenidos del Banco Mundial.

Un indicador alternativo es la entrada líquida de capitales como porcentaje del PIB. Pero esta variable es muy volátil y muy dependiente de los años considerados. Por es razón se optó por las reservas cambiarias como medida del colchón de liquidez en la economía.

7 Solamente en los modelos de efectos aleatorios se incluyeron variables dummy, que buscaban capturar posibles efectos regionales no vinculados a las variables anteriores. Fueron creadas dummies para Asia, África y América Latina, lo que implica que el valor del coeficiente de estas variables mide el efecto diferencial de pertenecer a una de estas regiones con relación a la tasa media de crecimiento de Europa y los Estados Unidos.

Gráfico 2_Correlación entre capacidad tecnológica y exportaciones de alta tecnología (2002)

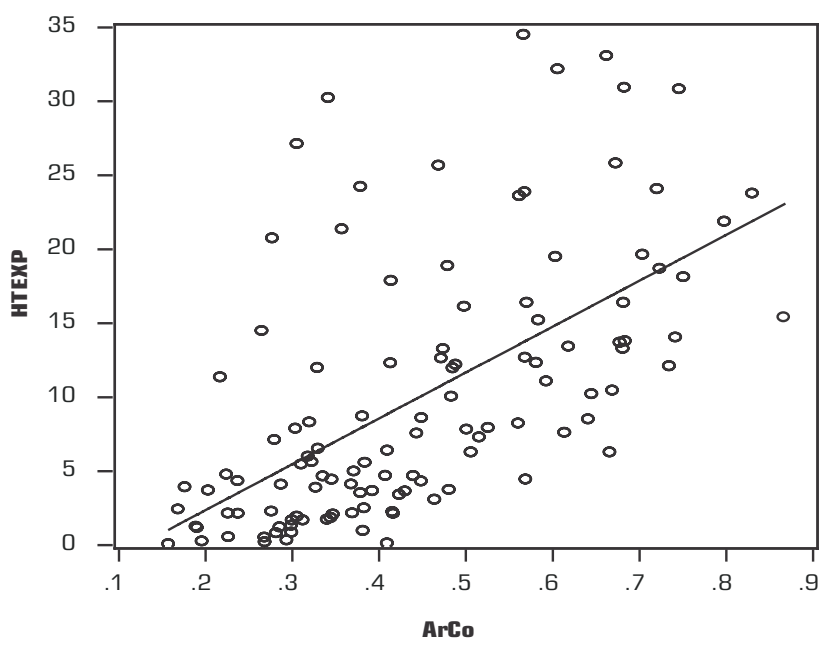

Fuente: World Bank (2006) y Archibugi y Coco (2003).

En los modelos con restricción de balanza de pagos la entrada de capitales puede ser una variable importante en el caso de los países en desarrollo

(McCombie y Thirlwall, 1994; cf. ecuación (6) más arriba). Por eso se incluyó, en el lado derecho de la ecuación, el valor de las reservas cambiarias (expresadas en términos de meses de importaciones de cada economía ${ }^{6}$. Se entiende que dicha variable es una buena proxy de la contribución de la entrada de capitales al alivio de la restricción externa sobre el crecimiento.

A la luz de las consideraciones anteriores, la ecuación estimada fue la siguiente:

$$
\begin{aligned}
\hat{y}_{u} & =\alpha_{i}+\beta_{0}+\delta_{0} d_{t}+\beta_{1} S_{i t} \\
& +\beta_{2} F_{i t}+\pi D_{R}+\varepsilon_{i t}
\end{aligned}
$$

donde $i$ denota el país, t denota el tiempo, $\hat{y}_{u}$ es la tasa de crecimiento del PIB per capita, $S_{*}$ es la participación de los sectores dinámicos en las exportaciones totales (medido por HTEXP, exportaciones de alta tecnología en relación a las exportaciones totales), $F_{\text {u }}$ son las reservas cambiarias en meses de importaciones, $D_{R}$ es un vector de variables dummy incluido en los modelos de efectos aleatorios, ${ }^{7}$ y $\varepsilon_{i t}$ es el término de error. Se usó una muestra de 59 
países para el período que transcurre entre 1990 y 2000. La variable $d_{t}$ es una variable dummy igual a cero cuando $t=1990$ e igual a uno cuando $t=2000$; ella no cambia según el país, razón por la cual no tiene el subscrito $i$. El intercepto de $t=1990$ es $\beta_{0}$ y el intercepto de $t=2000$ es $\beta_{0}+\delta_{0}$. La variable $\alpha_{i}$ capta todo los factores no observados que son constantes en el tiempo y que afectan $y_{i t}$.

El diseño econométrico tiene la forma de un panel de dos períodos. ${ }^{8}$ Esto permite controlar mejor la heterogeneidad existente entre los individuos por medio de una variable que capta efectos individuales que no son medidos por el econometrista, evitando problemas de variable omitida.

Hay dos modelos básicos de panel, el de efectos fijos y el de efectos aleatorios. El primero es una generalización del modelo constante-intercepto-inclinación, con variables dummy para los efectos de variables omitidas que permanecen constantes en el tiempo. En esta especificación, los efectos individuales pueden correlacionarse libremente con las demás variables explicativas. Se trata de una regresión múltiple con variables dummy para cada individuo, lo que hace que el intercepto de la regresión sea diferente para cada uno de ellos.

En el modelo de efectos aleatorios los efectos individuales son tratados como variables aleatorias. En él se asume que no hay correlación entre los efectos individuales y las otras variables explicativas, realizándose la estimación por medio de mínimos cuadrados generalizados.

Frees (2003) argumenta que si el propósito central es testar variables que permiten clasificar los individuos en grupos, el modelo de efectos aleatorios es más adecuado. Hsiao (1986, p. 42) observa que:

The fixed-effects model is viewed as one in which investigators make inferences conditional on the effects that are in the sample. The random-effects model is viewed as one in which investigators make unconditional or marginal inferences with respect to the population of all effect.

Algunas especificaciones de la ecuación (9) fueron estimadas tanto en modelos con efectos fijos como con efectos aleatorios. La adecuación de uno u otro modelo fue evaluada por medio de una prueba de Hausman. La hipótesis nula de esta prueba es que el modelo corresto es el de efectos fijos, mientras que la hipótesis alternativa es el de efectos aleatorios. Se examina el estadístico HS que tiende a cero bajo la hipótesis nula:
8 'Sobre las ventajas del uso

del Análisis de Panel con respecto a las regresiones cross-section convencionales ver Hsiao (1986). 


$$
H S=\left(b_{F E}-b_{R E}\right)\left[\operatorname{Var}\left(b_{F E}-b_{R E}\right)\right]^{-1}\left(b_{F E}-b_{R E}\right)
$$

donde $H S$ se distribuye $X^{2} \operatorname{con} K$ grados de libertad.

Obsérvese que el ejercicio econométrico propuesto es susceptible de sufrir problemas de causas endógenas. El modelo propone que los efectos de la tecnología sobre el crecimiento se dan sobre todo a través del patrón de especialización. Por esa razón, se recurrió a un procedimiento de estimación en dos etapas (LS2S) en que se usa la variable tecnológica como variable instrumental. Como la tecnología medida por el indicador ArCo no está correlacionada con el término de error y sí lo está con la especialización, dicho indicador puede desempeñar muy bien el papel de variable instrumental. ${ }^{9}$

El Cuadro 1 muestra los resultados del análisis de panel de dos períodos (1990 y 2000) donde la variable dependiente es la tasa de crecimiento del ingreso por habitante. Si bien fueron usadas distintas técnicas econométricas de estimación (pooled, panel con efectos fijos y panel con efectos aleatorios), solamente se presentan los resultados obtenidos con el modelo de efectos fijos (que se mostró superior al de efectos aleatorios de acuerdo a la prueba de Hausman). Los mismos sugieren que las predicciones del modelo cepalino- ricardiano son consistentes con la evidencia empírica ofrecida por la experiencia de crecimiento de la economía internacional en la década pasada.

En efecto, el Cuadro 1 muestra que las variables de especialización productiva mostraron una relación positiva con las tasas de crecimiento económico. La estimación con efectos fijos (columna 1d) revela que la especialización en productos intensivos en tecnología tiene un efecto más importante sobre el crecimiento que los términos de intercambio, o aún que el indicador de liquidez internacional empleado (reservas cambiarias definidas en meses de importaciones). Esas variables son significativas al 5\% cuando estimadas separadamente (columnas 1a, 1b y 1c), pero sólo al 10\% cuando se controla por la especialización del país en productos de alta tecnología.

Observando los resultados de las estimaciones en LS2S (columnas 2a-2d) se llega a las mismas conclusiones. Se nota además que los parámetros son mucho mayores que los obtenidos con las estimaciones LS de efectos fijos. Sin embargo, las reservas cambiarias no son estadísticamente significativas cuando la tecnología medida a través del indicador ArCo entra en la estimación como variable instrumental. 
Cuadro 1_Crecimiento y especialización externa: Análisis de Panel, años 1990 y 2000 - Variable Dependiente: Crecimiento del PIB por habitante

\begin{tabular}{|c|c|c|c|c|c|c|c|c|}
\hline & \multicolumn{4}{|c|}{ Efecto fijo 1/ } & \multicolumn{4}{|c|}{ LS2S 2/ } \\
\hline & (1a) & $(1 \mathrm{~b})$ & (1c) & (1d) & (2a) & (2b) & (2c) & (2d) \\
\hline ToT & $\begin{array}{l}0,24 * * \\
(0,098)\end{array}$ & & & $\begin{array}{c}0,21 * * * \\
(0,11)\end{array}$ & $\begin{array}{c}0,421^{* *} \\
(0,14)\end{array}$ & & & $\begin{array}{c}0,533 * * * \\
(0,289)\end{array}$ \\
\hline HTEXP & & $\begin{array}{c}0,0118^{* *} \\
(0,006)\end{array}$ & & $\begin{array}{c}0,355^{* *} \\
(0,121)\end{array}$ & & $\begin{array}{c}0,675^{* *} \\
(0,224)\end{array}$ & & $\begin{array}{c}0,754 * * \\
(0,301)\end{array}$ \\
\hline RESERVAS & & & $\begin{array}{c}0,013^{* *} \\
(0,005)\end{array}$ & $\begin{array}{c}0,095^{* * *} \\
(0,045)\end{array}$ & & & $\begin{array}{c}0,0129 \\
(0,0121)\end{array}$ & $\begin{array}{c}0,010 \\
(0,045)\end{array}$ \\
\hline $\mathrm{R} 2$ & 0,33 & 0,21 & 0,25 & 0,35 & 0,49 & 0,39 & 0,25 & 0,41 \\
\hline Individuos & 59 & 59 & 59 & 59 & 59 & 59 & 59 & 59 \\
\hline Observaciones & 118 & 118 & 118 & 118 & 118 & 118 & 118 & 118 \\
\hline
\end{tabular}

(**) significativo al $5 \%$;

$(* * *)$ significativo al $10 \%$

ToT = términos de intercambio;

HTEXP = porcentaje de las exportaciones de sectores de alta tecnología en el total de las exportaciones;

RESERVAS = las reservas cambiarias en meses de importaciones;

$1 /$ = Tests de Hausaman fueron obtenidos de las estimaciones de modelos de efectos aleatorios y todos indicaron que los modelos de efectos fijos son más adecuados;

2/ = La variable instrumental utilizada aquí en estos modelos estimados es el índice ArCo de capacidad tecnológica.

Fuente: Los autores a partir do World Development Indicatores, Word Bank (2006).

En resumen, procesos de convergencia y divergencia internacionales están en consonancia con procesos de convergencia y divergencia de las capacidades tecnológicas, que, a su vez, afectan el patrón de especialización. Otra forma de expresar esta misma conclusión es que la convergencia es condicional a un esfuerzo sostenido en las economías rezagas en el sentido de fortalecer sus capacidades de aprendizaje tecnológico, redefiniendo su inserción externa por medio de exportaciones en sectores más dinámicos.

\section{4_Comentarios finales}

El trabajo utilizó el modelo ricardiano con un continuo de bienes para analizar los vínculos entre brecha tecnológica, especialización y crecimiento. La estrategia adoptada para hacer el modelo fue la de suponer que la brecha afectaba el valor del intercepto de la 
curva $A$ de ventajas comparativas, y de esa manera, también afectaba el valor crítico $z_{r}$ de bienes producidos por el Sur. El modelo sugiere que la dinámica de la brecha podría sostener procesos de convergencia o de divergencia internacionales a partir de cambios en el patrón de especialización internacional del Sur.

El trabajo también testeó empíricamente el modelo para los años noventa, utilizando indicadores de competencia tecnológica y de dinamismo del patrón de especialización que aún no se habían explorado en modelos de este tipo. Los resultados fueron bastante satisfactorios, lo que sugiere que el modelo ricardiano Norte-Sur es compatible con la evidencia empírica disponible para los años noventa.

Se concluye que la especialización importa y que la competitividad auténtica (medida por la magnitud del esfuerzo tecnológico) juega un papel relevante en la definición de las tasas de crecimiento. Los años noventa representan un período en que la mayor parte de los países latinoamericanos buscó una nueva inserción en la economía internacional por medio de la liberalización comercial, sin que al mismo tiempo se diseñaran políticas tecnológicas activas capaces de inducir la transformación del patrón de especialización. Parece así confirmarse que el catching-up en términos de ingresos reales no puede disociarse de un esfuerzo de catching-up tecnológico. 


\section{Referencias Bibliográficas}

AMENDOLA, G.; DOSI, G.; PAPAGNI, G. The dynamics of international competitiveness. Welwirtschalftliches Arquiv: Band 129, Heft 3/93, p. 451-71, 1993

ARCHIBUGI, D.; COCO, A.

A new indicator of technological capabilities for developed and developing countries. First Globelics

Conference. Rio de Janeiro, 4-8 Nov. 2003.

AROCENA, R.; SUTZ, J. Learning divides, social capital and the role of the universities. First Globelics Conference. Rio de Janeiro, 4-8 Nov. 2003.

CIMOLI, M. Tecbnological gaps and institutional asymmetries in north-south model with a continuum of goods. Metroeconomica, n. 39, p. $245-274,1988$

CUTHBERSTSON, K.; HALL, S. G.; TAYLOR, M. Applied econometric techniques. New York: Philip Allan, 1992.

DOSI, G.; PAVITT, K.; SOETE, L. The economics of technical change and international trade. Brighton: Wheatsheaf, 1990.
DORNBUSH, R.; FISHER, S.; SAMUELSON, P. Comparative advantage, trade and payments in a ricardian model with a continuum of goods. American Economic Review, n. 67, p. 823-839, 1977.

FAGERBERG, J. Technology and international differences in growth rates. Journal of Economic Literature, n. 32, p. 1147-1175, 1994.

FAGERBERG, J. Technological progress, structural change and productivity growth: a comparative study. Structural Change and Economic Dynamics, n. 11, p. $393-411,2000$

FAJNZYLBER, F.

Industrialziación en América

Latina: de la Caja Negra al

Casillero Vacío. Santiago,

Cuadernos de la CEPAL, 1990.

FFRENCH-DAVIS, R

Macroeconomía, comercio y finanzas: para reformar las reformas en América Latina. Santiago: McGraw Hill, 2000.

FREES, E. W. 2003. Disponível em: <http://www.instruction. bus.wisc.edu/jfrees/gb806.htm>.
HSIAO, C. Analysis of Panel Data. Cambridge: UP, 1986.

(Econometrics Society

Monocraphs, n. 11).

McCOMBIE, J. S. L.; THIRLWALL, A. P. Economic growth and balance of payments constraint. New York: St Martin's Press, 1994.

NELSON, R e PACK, H.

The Asian miracle and modern growth theory. World Bank Working Papers Series, n. 1881, Oct. 1997.

OBSTFELD, M.; ROGOFF, K.

Foundations of international macroeconomics. MIT Press, 1996.

PREBISCH, R. Notes on Trade From the Standpoint of the Periphery. Cepal Review, n. 28, p. 203-214, Abr. 1986

REINERT, E. Competitiveness and its predecessors: a 500-year cross national perspective. Structural Change and Economic Dynamics, n. 6, p. 23-42, 1995. RODRÍGUEZ, O. La agenda del desarrollo: elementos para su discussion. In: BARBATO, C. Los grandes temas del desarrollo latinoamericano. Montevideo: Trilce, 2002.
ROMER, P. Endogenous technological change. Journal of Political economy, n. 98, (October): S71-S102, 1990.

ROS, J. Development theory and the economics of growth. Michigan University Press, 2000.

VERSPAGEN, B. Uneven growth between interdependent economies. Avebury: Aldershot, 1993.

WORLD BANK. World Development Indicators (on line). Washington, DC: World Bank, 2006.

Los autores agradecen las sugerencias de dos comentaristas : anónimos.

E-mail de contato do autor:

porcile@ufpr.br

Fecha de recepción: septiembre de 2005; fecha de aceptación: octubre de 2006. ..................... 